\title{
$i$-CONVEXITY OF MANIFOLDS WITH REAL PROJECTIVE STRUCTURES
}

\author{
SUHYOUNG CHOI
}

(Communicated by Christopher Croke)

\begin{abstract}
We compare the notion of higher-dimensional convexity, as defined by Carrière, for real projective manifolds with the existence of hemispheres. We show that if an $i$-convex real projective manifold $M$ of dimension $n$ for an integer $i$ with $0<i<n$ has an $i$-dimensional hemisphere, then $M$ is projectively homeomorphic to $\mathrm{S}^{n} / \Gamma$ where $\Gamma$ is a finite subgroup of $O(n+1, \mathbf{R})$ acting freely on $\mathbf{S}^{n}$.
\end{abstract}

A real projective structure (RP ${ }^{n}$-structure) on a smooth manifold of dimension $n, n>0$, is given by an atlas of charts to the sphere $\mathbf{S}^{n}$ where transition functions of the charts are restrictions of projective automorphisms of $\mathbf{S}^{n}$. An $\mathbf{R P}^{n}$-manifold is a manifold with an $\mathbf{R P}^{n}$-structure, and a projective map is an immersion-preserving real projective structures locally. The Klein model of hyperbolic geometry implies that $n$-dimensional hyperbolic manifolds provide examples of $\mathbf{R P}^{n}$-manifolds. (See [2] and [3].)

Throughout this paper, let $M$ be an $\mathbf{R P}^{n}$-manifold; let $\widetilde{M}$ denote the universal cover of $M$ with the induced $\mathbf{R P}^{n}$-structure. $M$ has a developing map $\operatorname{dev}: \widetilde{M} \rightarrow \mathbf{S}^{n}$, a projective map. The sphere $\mathbf{S}^{n}$ has a standard Riemannian metric $\mu$ of curvature 1 and the associated distance metric d. Note that dev induces a Riemannian metric $\mu$ on $\widetilde{M}$ from $\mathbf{S}^{n}$. Associated with $\mu$ is the induced distance metric d on $\widetilde{M}$. The completion $\check{M}$ of $\widetilde{M}$ is obtained by completing d. Let $\sigma$ be the frontier set $\check{M}-\widetilde{M}$. The sets $\check{M}$ and $\sigma$ are topologically independent of the choice of $\mathrm{dev}$, and dev extends uniquely to a distance decreasing map on $\check{M}$. The extended map is also called a developing map and is denoted by the same symbol dev .

Let $i$ be an integer such that $0<i \leq n$ holds. A great $i$-sphere is a totally geodesic $i$-dimensional sphere imbedded in $\mathbf{S}^{n}$; a subset of $\check{M}$ for which the restriction of $\operatorname{dev}$ is an imbedding onto a great $i$-sphere in $\mathbf{S}^{n}$ is also called a great $i$-sphere. A great $i$-ball is a hemisphere of a great $i$-sphere $\mathbf{S}^{i}$ in $\mathbf{S}^{n}$; a

Received by the editors August 23, 1991 and, in revised form, January 8, 1993.

1991 Mathematics Subject Classification. Primary 53C10, 53C15, 57M50; Secondary 51A05, 52A01.

Key words and phrases. Convexity, generalization of convexity, projective geometry, geometric structures, real projective structures.

The author gratefully acknowledges partial support from TGRC-KOSEF. 
subset of $\check{M}$ for which the restriction of dev is an imbedding onto a great $i$ ball is also called a great $i$-ball. An $n$-dimensional open hemisphere of $\mathbf{S}^{n}$ has a natural affine structure. An $i$-simplex is the convex hull of $i+1$ independent points in the hemisphere (under affine geometry). An $i$-simplex in $\check{M}$ is a subset for which the restriction of $\mathrm{dev}$ is an imbedding onto an $i$-simplex in an $n$-dimensional open hemisphere of $\mathbf{S}^{n}$.

We introduce the definition given by Carrière [1]. We say that $M$ is $i$-convex for an integer $i$ with $0<i<n$ if the following holds: Given an $(i+1)$-simplex $T$ in $\check{M}$, if $F_{1}$ is a face of $T$ such that $T \cap \sigma=F_{1}^{o} \cap \sigma$, then $T \subset \widetilde{M}$. (Note that the $i$-convexity of $M$ implies the $j$-convexity of $M$ where $i \leq j<n$.)

A subset $A$ of $\check{M}$ is called convex if every two points of $A$ are connected by an imbedded arc $\alpha$ such that $\operatorname{dev} \mid \alpha$ is an imbedding onto a segment of d-length $\leq \pi$ (see $[2, \S 1]$ for more detail). It is easy to see that given a convex open subset $A$ of $\widetilde{M}$, the map $\operatorname{dev} \mid A$ is isometric with respect to the metrics on $\widetilde{M}$ and $\mathrm{S}^{n}$. Thus, $\operatorname{dev} \mid \mathrm{Cl}(A)$ is an imbedding onto $\mathrm{Cl}(\operatorname{dev}(A))$ where $\mathrm{Cl}(A)$ is the closure of $A$ in $\check{M}$.

We prove the following theorem in this paper.

Theorem. Suppose that $M$ is $i$-convex where $0<i<n$ holds. Suppose that $\widetilde{M}$ includes a great $j$-ball or a great $j$-sphere for $i \leq j<n$. Then $\widetilde{M}$ is projectively homeomorphic to $\mathbf{S}^{n}$.

The conclusion implies that $M$ is projectively homeomorphic to $\mathbf{S}^{n} / \Gamma^{\prime}$ where $\Gamma^{\prime}$ is a subgroup of the projective automorphism group $\operatorname{Aut}\left(\mathbf{S}^{n}\right)$ acting freely and properly discontinuously on $\mathbf{S}^{n}$. It is a standard fact that $\Gamma^{\prime}$ is conjugate to a finite subgroup of $O(n+1, \mathbf{R})$, the group of isometries of $\mathbf{S}^{n}$. Thus, $M$ is projectively homeomorphic to $\mathbf{S}^{n} / \Gamma$ where $\Gamma$ is a finite subgroup of $O(n+1, \mathbf{R})$ acting freely on $\mathbf{S}^{n}$.

Proof of the Theorem. We give the proof of the theorem assuming that Lemma 1 , which follows, holds. We can prove the theorem by using induction on $j$. Suppose that $j=n-1$. Then by Lemma $1 \widetilde{M}$ includes a great sphere of dimension $n$. Therefore, $\widetilde{M}$ is projectively homeomorphic to $\mathbf{S}^{n}$.

Suppose that the conclusion is true for the case where $j=k>i$ holds. We verify the conclusion for the case where $j=k-1 \geq i$ holds. By Lemma 1 , $\widetilde{M}$ includes a great $k$-sphere. By the induction hypothesis, $\widetilde{M}$ is projectively homeomorphic to $\mathbf{S}^{n}$. This completes the proof.

Let us discuss Lemma 1. For an integer $j$ with $0<j<n, \mathrm{a}(j+1)$-bihedron in $\mathbf{S}^{n}$ is a closed domain in a great $(j+1)$-sphere $\mathbf{S}^{j+1}$ in $\mathbf{S}^{n}$ bounded by two great $j$-balls with common boundary equal to a great $(j-1)$-sphere or the set of two points antipodal to each other; a $(j+1)$-bihedron in $\check{M}$ is a subset for which the restriction of dev is an imbedding onto a $(j+1)$-bihedron in $\mathbf{S}^{n}$. The bounding great $j$-balls of a bihedron are called faces. A bihedron in $\mathbf{S}^{n}$ or $\widetilde{M}$ is convex if and only if the interior angle between two faces of the bihedron is less than or equal to $\pi$.

Lemma 1. Assume that $M$ is $i$-convex for an integer $i$ with $0<i<n$. Suppose that $\widetilde{M}$ includes a great $j$-ball $B_{0}$ where $i \leq j<n$. Then $\widetilde{M}$ includes a great $(j+1)$-sphere. 
Proof. We choose a convex $(j+1)$-bihedron $T_{0}$ including $B_{0}$ in a neighborhood of $B_{0}$ with faces $B_{a}$ and $B_{b}$ such that $\delta B_{a}=\delta B_{b}=\delta B_{0}$ holds. (Assume that $B_{0}$ is not a face of $T_{0}$.) Let us agree that bihedra in this proof are $(j+1)$ dimensional always. Let $A^{+}$be the set of convex bihedra with a face $B_{0}$ and including $B_{a}$, and let $A^{-}$be the set of convex bihedra with a face $B_{0}$ and including $B_{b}$. Then there is a unique great $(j+1)$-sphere $\mathbf{S}^{j+1}$ in $\mathbf{S}^{n}$ including the images of elements of $A^{+}$and $A^{-}$under dev.

We may parameterize $A^{+}$and $A^{-}$by positive intervals. Given an element $T$ of $A^{+}$or $A^{-}$, let $\theta(T)$ be the interior angle between $B_{0}$ and the other face of $T$. This defines a function $\theta$ from the set of elements of $A^{+}$and $A^{-}$to $\mathbf{R}$. Let $T_{a}$ be the bihedron in $T_{0}$ bounded by $B_{0}$ and $B_{a}$; let $T_{b}$ be the bihedron in $T_{0}$ bounded by $B_{0}$ and $B_{b}$. Suppose that $T^{\prime}$ and $T^{\prime \prime}$ are two bihedra in $A^{+}$with $\theta\left(T^{\prime}\right)=\theta\left(T^{\prime \prime}\right)$. Then Lemma 2 implies that $T^{\prime}=T^{\prime \prime}$. Thus $\theta \mid A^{+}$ is an injective map into $\left[\theta\left(T_{a}\right), \pi\right]$. Similarly, $\theta \mid A^{-}$is an injective map into $\left[\theta\left(T_{b}\right), \pi\right]$.

If we have $t<t^{\prime}$ where $t^{\prime} \in \theta\left(A^{+}\right)$and $t \in\left[\theta\left(T_{a}\right), \pi\right]$ hold, then $t$ is realized as the angle of a bihedron in $A^{+}$which is included in the bihedron corresponding to $t^{\prime}$. It follows that $\theta\left(A^{+}\right)$is connected. Similarly, $\theta\left(A^{-}\right)$is connected.

Let $T \in A^{+}$. Then $\operatorname{dev} \mid T$ is an imbedding onto a convex bihedron $\operatorname{dev}(T)$. Choose an open neighborhood $N$ of $T$ in $\widetilde{M}$ such that $\operatorname{dev} \mid N$ is an imbedding onto an open subset of $\mathbf{S}^{n}$. Then for every convex bihedron $T^{\prime}$ in $\operatorname{dev}(N)$ the open subset $N$ includes a convex bihedron $T^{\prime \prime}$ such that $\operatorname{dev}\left(T^{\prime \prime}\right)=T^{\prime}$. This implies that $\left[\theta\left(T_{a}\right), \pi\right]$ includes an open neighborhood of $\theta(T)$ whose elements are realized by bihedra in $A^{+}$. Hence, $\theta\left(A^{+}\right)$is an open subset of $\left[\theta\left(T_{a}\right), \pi\right]$. Similarly, $\theta\left(A^{-}\right)$is an open subset of $\left[\theta\left(T_{b}\right), \pi\right]$.

We claim that $\theta\left(A^{+}\right)$is closed. (We use $i$-convexity now.) Suppose that it is not closed. Then $\theta\left(A^{+}\right)$is the half-open interval $\left[\theta\left(T_{a}\right), t^{+}\right)$for a real number $t^{+}$less than or equal to $\pi$. Let $T_{1}=\bigcup_{T \in A^{+}} T^{o}$. Since by Lemma $2, \operatorname{dev} \mid T_{1}$ is injective, $\operatorname{dev} \mid T_{1}$ is an imbedding onto the interior of a convex bihedron of angle $t^{+}$. For the closure $\mathrm{Cl}\left(T_{1}\right)$ of $T_{1}$ in $\check{M}$, the map $\operatorname{dev} \mid \mathrm{Cl}\left(T_{1}\right)$ is an imbedding onto $\mathrm{Cl}\left(\operatorname{dev}\left(T_{1}\right)\right)$. Since $\mathrm{Cl}\left(\operatorname{dev}\left(T_{1}\right)\right)$ is a convex bihedron, so is $\mathrm{Cl}\left(T_{1}\right)$. The bihedron $\mathrm{Cl}\left(T_{1}\right)$ has two faces $B_{0}$ and $B_{\mathrm{Cl}\left(T_{1}\right)}$ where $\sigma \cap \mathrm{Cl}\left(T_{1}\right) \subset$ $B_{\mathrm{Cl}\left(T_{1}\right)}^{o}$. Since $\sigma \cap B_{\mathrm{Cl}\left(T_{1}\right)}$ is compact, $\sigma \cap B_{\mathrm{Cl}\left(T_{1}\right)}^{o}$ is a bounded subset of the open great ball $B_{\mathrm{Cl}\left(T_{1}\right)}^{o}$. Thus, the bihedron $\mathrm{Cl}\left(T_{1}\right)$ includes a $(j+1)$-simplex $K$ such that $\sigma \cap \mathrm{Cl}\left(T_{1}\right) \subset K_{1}^{o}$ where $K_{1}$ is a $j$-dimensional face of $K$ and is included in $B_{\mathrm{Cl}\left(T_{1}\right)}$. The definition of $i$-convexity implies that $K_{1} \cap \sigma=\varnothing$ and $\mathrm{Cl}\left(T_{1}\right) \subset \widetilde{M}$ hold. Since $\mathrm{Cl}\left(T_{1}\right) \supset T_{a}$, we have $\mathrm{Cl}\left(T_{1}\right) \in A^{+}$and hence $t^{+} \in \theta\left(A^{+}\right)$. This is absurd. Therefore, $\theta\left(A^{+}\right)$and, similarly, $\theta\left(A^{-}\right)$are closed.

We therefore have $\theta\left(A^{+}\right)=\left[\theta\left(T_{a}\right), \pi\right]$ and $\theta\left(A^{-}\right)=\left[\theta\left(T_{b}\right), \pi\right]$. Let $T^{+}$be the convex bihedron corresponding to $\pi$ belonging to $A^{+}$, and let $T^{-}$be that belonging to $A^{-}$. We have $T^{+}, T^{-} \subset \widetilde{M}$. The maps $\operatorname{dev} \mid T^{+}$and $\operatorname{dev} \mid T^{-}$ are imbeddings onto great $(j+1)$-balls in the great $(j+1)$-sphere $\mathbf{S}^{j+1}$. Since the intersection $\operatorname{dev}\left(T^{+}\right) \cap \operatorname{dev}\left(T^{-}\right)$is a great $j$-sphere and, hence, is pathconnected, Lemma 2 implies that $\operatorname{dev} \mid T^{+} \cup T^{-}$is an imbedding onto $\mathbf{S}^{j+1}$. Therefore, $T^{+} \cup T^{-}$is a great $(j+1)$-sphere in $\widetilde{M}$. This completes the proof of Lemma 1. 
Lemma 2. Suppose that $A$ and $B$ are path-connected compact subsets of $\widetilde{M}$ such that $\operatorname{dev} \mid A$ and $\operatorname{dev} \mid B$ are imbeddings. Suppose that $A \cap B \neq \varnothing$ and that $\operatorname{dev}(A) \cap \operatorname{dev}(B)$ is a path-connected subset of $\mathbf{S}^{n}$.

Then $\operatorname{dev} \mid A \cup B$ is an imbedding onto $\operatorname{dev}(A) \cup \operatorname{dev}(B)$.

Proof. We only need to deduce the injectivity of $\operatorname{dev} \mid A \cup B$ from the fact that given a path in $\mathbf{S}^{n}$ and an initial point in $\widetilde{M}$ there is at most one lift of the path to $\widetilde{M}$ (see [1, Proposition 1.3.1]).

Let us end this paper with the following remark: Carrière conjectured in 1988 that the homotopy groups in dimensions greater than or equal to $i, i>1$, for an $i$-convex affine manifold are trivial. This conjecture is still open. What we did here may aid us in understanding similar questions for projective manifolds.

\section{REFERENCES}

1. Y. Carrière, Autour de la conjecture de L. Markus sur les variétés affines, Invent. Math. 95 (1989), 615-628.

2. S. Choi, Convex decompositions of real projective surfaces I: $\pi$-annuli and convexity (to appear).

3. D. Sullivan and W. Thurston, Manifolds with canonical coordinate charts: some examples, Enseign. Math. (2) 29 (1983), 15-25.

Topology and Geometry Research Center, Kyungpook National University, 702-701 TAEgU, SOUTH KOREA 